Annuaire suisse de politique de développement

9 | 1990

Annuaire Suisse - Tiers Monde 1990

\title{
Fuite de capitaux et Tiers Monde : un commentaire
}

\section{Guido Pult}

\section{OpenEdition}

\section{Journals}

Édition électronique

URL : http://journals.openedition.org/aspd/1321

DOI : 10.4000/aspd.1321

ISSN : 1663-9669

\section{Éditeur}

Institut de hautes études internationales et du développement

\section{Édition imprimée}

Date de publication : 1 janvier 1990

Pagination : 193-198

ISSN : 1660-5934

\section{Référence électronique}

Guido Pult, «Fuite de capitaux et Tiers Monde : un commentaire », Annuaire suisse de politique de développement [En ligne], 9 | 1990, mis en ligne le 28 mars 2013, consulté le 08 septembre 2020. URL : http://journals.openedition.org/aspd/1321 ; DOI : https://doi.org/10.4000/aspd.1321 


\title{
Fuite de capitaux et Tiers Monde: un commentaire
}

\author{
Guido Pult
}

1. Le lecteur des articles de Thobias Bauer et de Hans J. Mast risque d'être déconcerté surtout par l'énorme différence de leurs estimations et ce n'est que de cet aspect de la controverse que nous traiterons dans cette note.

Pour Bauer la fuite de capitaux en Suisse à partir du Tiers Monde porterait sur 250-300 milliards de francs (valeur cumulée, c'est-à-dire stock, en 1988) ce qui, d'après lui, correspondrait aux 25-30\% du total. A l'opposé Mast finit par conclure que le phénomène est pratiquement inexistant. Ainsi en passant de la première estimation à la deuxième c'est un montant de l'ordre de grandeur du produit national suisse qui s'envole.

Malgré ces différences, il est possible de trouver un point commun à ces deux approches, un point à partir duquel les deux auteurs, en prenant des directions opposées, se sont éloignés l'un de l'autre. Il s'agit du montant des engagements totaux (opérations fiduciaires comprises) des banques suisses à l'égard de clients du Tiers Monde: 164 milliards de francs en 1988. Ce montant qui est présent dans le texte de Bauer correspond en gros - en considérant aussi les pays pétroliers et la situation d'avant 1977 et de 1988 - à la valeur cumulée des variations annuelles des mêmes engagements sur lesquelles Mast fonde ses estimations.

Je remercie Tobias Bauer et Hans J. Mast des remarques qu'ils ont eu la gentillesse de me transmettre. 
2. L'élément essentiel qui permet à Bauer d'arriver à un montant d'environ 300 milliards est représenté par la prise en compte de la valeur des titres que les banques suisses administrent pour le compte de clients du Tiers Monde. Afin d'évaluer cet élément - sur lequel il n'y a pas de statistiques officielles - Bauer s'appuie sur une étude de la firme Mac Kinsey, étude qui pour être fortement subjective jouit néanmoins, de par sa signature, d'une autorité qui en justifie l'utilisation. Par ailleurs, l'opportunité d'inclure les titres dans le calcul est soulignée aussi dans le texte de Mast.

Ce qui dans cette démarche prête à discussion concerne d'abord les pays inclus dans le Tiers Monde. En effet, Bauer aboutit à une somme d'environ 300 milliards de francs en calculant le $20 \%$ - ce qui serait la proportion des placements revenant au Tiers Monde - des placements totaux en Suisse. Mais chez Mac Kinsey, cette proportion se rapporte en réalité à l'ensemble du monde exceptés les pays européens et les USA. Elle couvre donc également des pays tels que le Japon, l'Afrique du Sud et les pays pétroliers du Moyen-Orient pour lesquels les placements à l'étranger ne sont pas considérés comme de la fuite de capitaux. En utilisant comme clef de répartition la structure des placements (titres exclus) selon la statistique publiée par la BNS, la proportion utilisée par Bauer doit donc être diminuée d'environ un tiers. L'estimation de 250-300 milliards se réduit ainsi à 170-200 milliards de francs.

Une révision semblable doit être conduite aussi à l'égard de la part aboutissant en Suisse de la fuite totale de capitaux. Part que Bauer, on l'a vu, fixe à 25$30 \%$. Ce chiffre est obtenu en rapportant les 250-300 milliards d'avant au total des avoirs détenus à l'extérieur par le Tiers Monde tel qu'il est estimé par Deppler-Williamson (1987). Or le montant figurant dans cette étude, contrairement au premier montant, d'un cóté exclut les pays pétroliers du Moyen-Orient, et de l'autre comprend aussi les placements du secteur public. En homogénéisant les deux grandeurs et en se référant à l'année du calcul de Deppler-Williamson, c'est-à-dire 1985 , la proportion recherchée par Bauer se réduit de $25-30 \%$ à environ $20 \%$.

3. Quel sens faut-il alors attribuer à l'estimation de 170-200 milliards à laquelle on parvient à partir de l'article de Bauer? S'agit-il, comme cet auteur le suggère, presque entièrement de fuite de capitaux? II est bien évident que les avoirs détenus à l'extérieur par un pays relèvent aussi d'autres motivations. Parmi celles-ci figurent en particulier le renforcement de la capacité productive (peu importe si elle a lieu uniquement à l'étranger) ou commerciale des entreprises nationales ou encore le développement des affaires internationales des banques ou la diversification internationale des portefeuilles. Selon DepplerWilliamson, pour le Tiers Monde (défini comme lors des calculs ci-dessus), la proportion des avoirs à l'extérieur expliquée par ces motivations "normales" est de l'ordre de 2/5èmes. En utilisant cette proportion, la valeur des capitaux en fuite vers la Suisse devient d'environ 100-120 milliards. La prise en compte de la motivation "normale" - puisqu'elle s'applique aussi au dénominateur - n'a 
par contre pas d'effet sur la part échéant à la Suisse dans le total de la fuite des capitaux.

II s'agit par là de ce qu'on peut dire à propos de la partie visible de la fuite des capitaux. Car pour être complet il faudrait encore ajouter les capitaux qui parviennent en Suisse par des intermédiaires résidents dans les pays industrialisés (en particulier dans notre pays) et les placements en avoirs non financiers (notamment en immeubles et terrains).

4. Voyons maintenant comment chez Mast la fuite de capitaux devient quantité négligeable. Cet auteur n'envisage que la statistique des engagements des banques suisses, donc sans considérer les titres et sans séparer les flux privés des flux publics. Ses calculs se rapportent aux flux de la période 1976-87. Mast exclut, correctement comme on l'a vu, les pays pétroliers du Moyen-Orient. Toutefois, puisque les statistiques sur lesquelles ils se fondent - qui sont celles de la BNS - ne le lui permettent pas, à la différence de Bauer, il renonce à déduire les capitaux provenant des centres off-shore des Caraïbes dont l'origine n'est pas le Tiers Monde. Ce qu'il y a de particulier dans sa démarche c'est d'abord le fait qu'il biffe les intérêts payés par nos banques: ces derniers étant d'origine suisse, ils ne feraient pas partie des capitaux en fuite. Cette manière de procéder, qui se justifie dans le cadre de certaines comparaisons traditionnelles, laisse perplexe, car elle ne correspond pas au concept de fuite de capitaux utilisé à présent et le plus souvent par les organisations internationales. En particulier elle n'est pas conforme à l'idée, très répandue dans les publications récentes du FMI, que la fuite de capitaux est mesurée par la différence entre les avoirs totaux à l'étranger et les avoirs qu'on obtient en capitalisant les revenus déclarés du capital à l'étranger. Remarquons qu'après la déduction en question, Mast - pendant un instant - estime que la part des capitaux en fuite se dirigeant vers l'étranger est de l'ordre de $8-10 \%$. Si pour faciliter les comparaisons avec le texte de Bauer on convertit les flux en stocks, on aboutit en réintégrant les intérêts à une proportion d'environ 12\%. Rappelons qu'avant de comparer cette estimation à celle de $20 \%$ que nous avons effectuée à partir du texte de Bauer, il faudrait isoler les capitaux privés et surtout déduire les capitaux off-shore provenant des pays industrialisés et ajouter la valeur des titres. Eu égard à l'ordre de grandeur possible de ces différents éléments (en particulier s'il est vrai, comme l'affirme le rapport Mc Kinsey, que les titres représentent les 2/3 du total des avoirs gérés par les banques suisses) on ne peut pas exclure que pour lesdits aménagements les deux estimations, qui déjà ne sont pas très éloignées, se rapprochent considérablement.

Mast toutefois ne s'arrête pas là: pour lui la proportion trouvée ne correspond nullement à de la fuite de capitaux car l'arrivée en Suisse de capitaux du Tiers Monde serait presque entièrement expliquée par la différence entre taux d'intérêt réels. De cette manière Mast met l'accent sur un facteur explicatif fondamental. Toutefois l'idée que ce facteur exclut le phénomène de la fuite n'est pas conforme aux usages. Nous reviendrons sur ce point plus loin, à l'aide des quelques compléments ci-après. 
5. Nous nous permettrons d'abord d'ajouter quelques données concernant la relation entre d'une part les engagements des banques suisses selon la statistique de la BNS et d'autre part la fuite de capitaux vers l'étranger selon les experts du FMI. Les limites des statistiques disponibles nous ont poussé à ne retenir que deux pays: l'Argentine et le Brésil. Comme le montrent les figures 1 et 2, il y a entre les deux courbes un parallélisme assez net. Dans le cas de l'Argentine on remarque en particulier que la pointe de 1982 concernant la fuite totale de capitaux se retrouve aussi dans les chiffres suisses. Pour le Brésil, à partir de 1979, le parallélisme est encore plus frappant. Le nombre peu élevé d'observations empêche une analyse statistique approfondie. Mais il est suffisant pour renforcer sensiblement la confiance dans les chiffres publiés par la BNS, du moins en regard des comparaisons internationales.

6. Les graphiques en question laissent aussi supposer que la fuite de capitaux en Suisse et la fuite totale sont expliquées dans une large mesure par les mêmes causes. C'est parmi ces causes que le différentiel des taux d'intérêt réel évoqué par Mast a joué un rôle déterminant. En effet, derrière l'évolution de ce différentiel se cache celle des taux d'inflation et le lien est ainsi établi avec ce qui dans la littérature récente est considéré comme le mécanisme fondamental de la fuite des capitaux pour les années 70-80. Sur la base notamment de Sachs (1989) ce mécanisme, en réduisant l'explication au minimum, peut être précisé dans les termes suivants: d'un côté les dépenses des gouvernements d'importants pays du Tiers Monde ont fortement augmenté, notamment en Amérique latine, sous la poussée de revendications des groupes sociaux les mieux organisés et parfois aussi de "prédateurs", dont Marcos ne représente qu'un exemple extrême; de l'autre côté l'accroissement des recettes a été freiné par le souci de ne pas heurter ceux qui auraient dû passer à la caisse. Le déficit qui en est résulté a été largement financé par l'accroissement de la masse monétaire, ce qui a maintenu les taux d'intérêts nominaux relativement bas et accéléré l'inflation. Cela s'est traduit par des taux d'intérêts réels négatifs et en même temps par des taux de change surévalués (d'où la crainte de dévaluations), c'est-à-dire par ce que Paul Krugman (1989) appelle "les deux causes structurelles" de la fuite des capitaux pour les années en question.

La relation, souvent mal comprise, entre fuite de capitaux et endettement s'explique aisément dans le même contexte: l'endettement étant dans cette relation le moyen par lequel les banques centrales se sont procuré les devises pour défendre le taux de change contre les attaques provenant de la fuite de capitaux.

Naturellement à l'intérieur d'un tel mécanisme il reste à expliquer le pourquoi du rôle privilégié joué par la Suisse. Mais sur ce point les deux auteurs sont proches l'un de l'autre et nous n'y reviendrons pas.

7. Il est évident que le mécanisme que nous venons de préciser n'explique pas tout. Car faute de statistiques, trop d'exemples prouvent qu'une partie des capitaux qui arrivent en Suisse depuis le Tiers Monde est d'origine criminelle (no- 
Figure 1

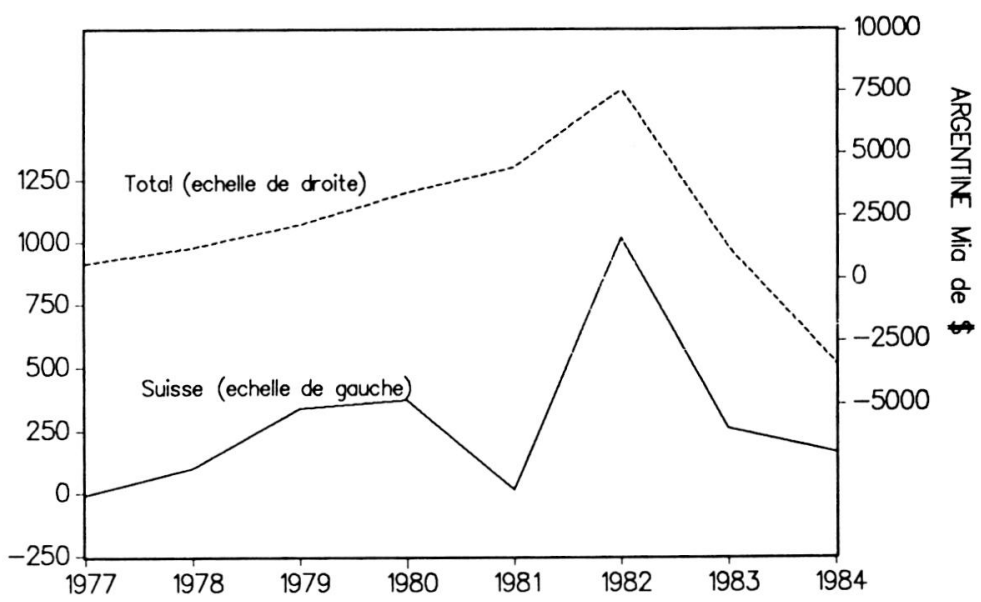

- Variation annuelle des engagements des banques suissses à l'égard de l'Argentine, Sources : BNS.

-.-----Variation annuelle des capitaux totaux en fuite depuis I'Argentine, Source : Cumby-Levich (1987); méthode Cine

\section{Figure 2}

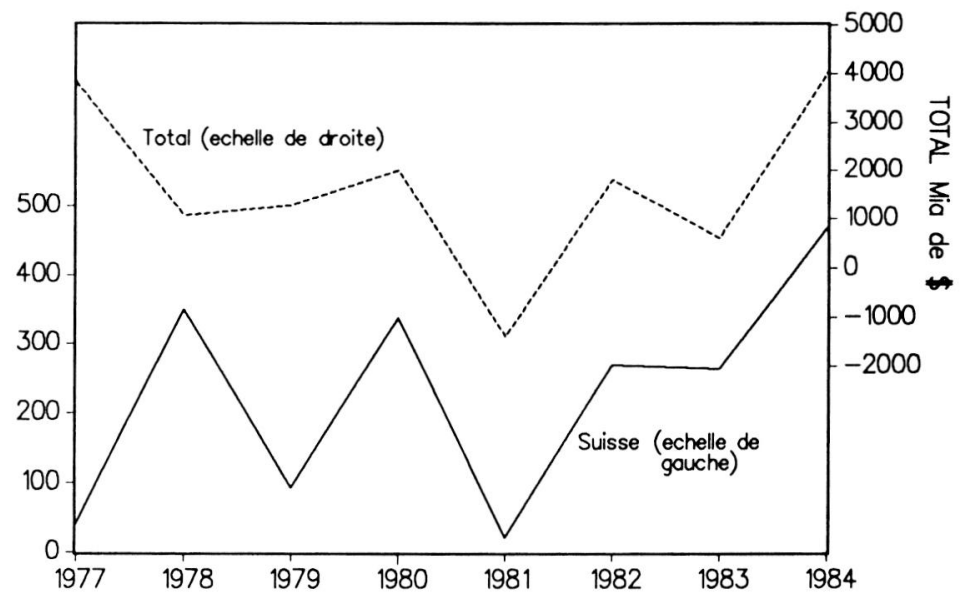

Variation annuelle des engagements des banques suissses à l'égard du Brésil, Sources : BNS.

- Variaiton annuelle des capitaux totaux en fuite depuis le Brésil, Source : Cumby-Levich (1987); méthode Cine 
tamment lors d'opérations de blanchiment) ou a comme but l'évasion fiscale. Or à l'évidence c'est essentiellement par rapport à ces demières catégories de capitaux que le sens de responsabilité de nos banques peut être mis en question et que notre législation peut être jugée comme inadéquate. Mast, étant convaincu que la proportion d'origine criminelle est somme toute négligeable, n'examine que la composante purement économique des capitaux en fuite: ce qui automatiquement provoque l'impression que nos banquiers sont innocents et que nos lois ne doivent pas être modifiées. A l'opposé chez Bauer l'argumentation et encore plus le ton relèvent souvent d'une attitude d'incrimination qu'on ne peut admettre que par rapport aux fuites de nature illégale. On l'aura compris, il s'agit de deux points de vue trop éloignés l'un de l'autre pour que la lecture d'un texte dispense de celle de l'autre.

\section{Références}

- Cumby R. et Levich R., On the Definition and Magnitude of recent capital flight, in J. Williamson, Capital Flight and Third World Debt, Institute for international economics, 1987

- Deppler M. et Williamson M., Capital Flight: Concepts, Measurement, and Issues, in IMF, Staff Studies for the World Economic Outlook, Washington, August 1987

- Krugman P., Private Capital Flows to Problem Debtors, in Developing Country Debt and Economic Performance, J.D. Sachs editor, The University of Chicago Press, 1989

- Sachs J.D., Introduction, in Developing Country Debt and Economic Performance, J.D. Sachs ed., The University of Chicago Press, 1989 